decreased from $186 \pm 68 \mathrm{ml}$ to $157 \pm 68 \mathrm{ml}(\mathrm{p}<0.001) .18 \quad(56 \%)$ patients exhibited RR. There was a significant relationship between percentage rise in $\mathrm{LV}-\mathrm{dP} / \mathrm{dtmax}$ and $\mathrm{RR}$ for DDDLV pacing $(p<0.001)$ (Abstract 90 figure 2). A similar relationship for AHR and RR in DCM and ICM $(p=0.01 \& p=0.006)$ was seen.

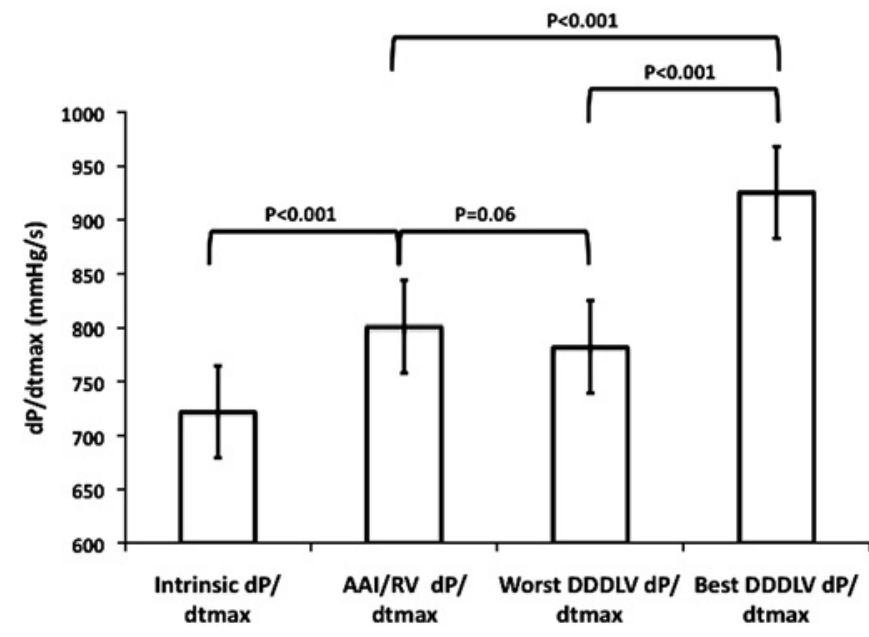

Abstract 90 Figure 1

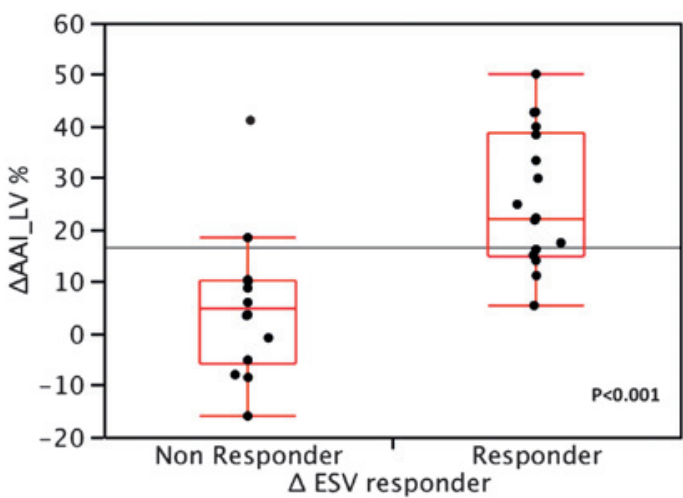

Abstract 90 Figure 2

Conclusions Acute haemodynamic response to LV pacing is useful for predicting which patients are likely to remodel in response to CRT both for DCM and ICM. There is much variation in the rise in LV-dP/dtmax depending on LV lead position. Using acute haemodynamic response measured with a pressure wire during CRT implant has the potential to guide LV lead positioning and improve response rates in the future.

\section{RIGHT VENTRICULAR DYSFUNCTION IDENTIFIES CLINICAL OUTCOMES FOLLOWING CARDIAC RESYNCHRONISATION THERAPY}

doi:10.1136/heartjnl-2011-300198.91

${ }^{1} \mathrm{~K}$ Guha, ${ }^{2} \mathrm{~F}$ Alpendurada, ${ }^{2} \mathrm{~S}$ Prasad, ${ }^{2} \mathrm{~T}$ McDonagh, ${ }^{1} \mathrm{M}$ R Cowie, ${ }^{2} \mathrm{R}$ Sharma. ${ }^{1}$ Royal Brompton Hospital, Imperial College, London, UK; ${ }^{2}$ Royal Brompton Hospital, London, UK

Background Cardiac resynchronisation therapy (CRT) is an established treatment for patients with advanced heart failure (HF). However, a proportion of patients do not derive benefit post implantation of CRT. Despite an established predictive role in HF, the significance of RV dysfunction in gauging clinical benefit from
CRT has not been investigated. Cardiovascular magnetic resonance (CMR) is an important tool in the assessment of HF and is considered the gold-standard in estimating RV function. We used this technique to assess the impact of RV dysfunction on clinical outcomes following CRT implantation.

Methods We evaluated 48 consecutive patients attending a heart failure pacing clinic who had a CMR study within 6 months prior to CRT implantation. Clinical, biochemical, ECG and imaging data were collected. Biventricular function and myocardial scar were assessed by CMR including gadolinium enhancement. The primary end-point was a composite of all cause mortality (ACM) or unplanned cardiovascular hospitalisation.

Results The mean age was $64.5 \pm 12.7$ years. HF was ischaemic in $42 \%$ of patients, and $85 \%$ were in NYHA class III/IV at the time of implantation. Atrial fibrillation/flutter was found in $27 \%$ of patients. The mean LVEF estimated by CMR was $27 \pm 8 \%$, while the median RVEF was 52\% (IOR 35\%-63\%). The mean tricuspid annular plane systolic excursion (TAPSE) was $14.0 \pm 6.0 \mathrm{~mm}$, and the mean pulmonary artery pressure (on echocardiography) was 37 $\pm 10 \mathrm{~mm} \mathrm{Hg}$. Ten patients $(21 \%)$ met the primary end-point over a mean follow-up of 28.6 months. On time-to-event analysis, only atrial fibrillation (HR 4.8, $\mathrm{p}=0.02$ ) and RV dysfunction, ie, reduced RVEF (HR 0.96 per 1\% EF, $\mathrm{p}=0.01$ ) or TAPSE (HR 0.80 per mm, $\mathrm{p}<0.01)$ were independent predictors of the primary end-point. Atrial fibrillation and low RVEF were the only independent predictors of mortality ( $p=0.03$ and 0.04 , respectively).

Conclusions RV dysfunction is an independent predictor of adverse clinical outcomes following CRT. The assessment of RV function may be considered in patient selection for CRT implantation.

\section{IDENTIFYING PATIENTS WITH CHRONIC HEART FAILURE: A COMPARISON OF THE GOLD STANDARDS FRAMEWORK WITH A CLINICAL PROGNOSTIC MODEL}

doi:10.1136/heartjnl-2011-300198.92

${ }^{1} \mathrm{~K}$ K Haga, ${ }^{2} \mathrm{~S}$ A Murray, ${ }^{3} \mathrm{~J}$ Reid, ${ }^{3} \mathrm{~A}$ Ness, ${ }^{3} \mathrm{M}$ O'Donnell, ${ }^{4} \mathrm{D}$ Yellowlees, ${ }^{5} \mathrm{M}$ A Denvir ${ }^{1}$ University of Edinburgh, School of Medicine and Veterinary Medicine, Edinburgh, UK; ${ }^{2}$ Primary Palliative Care Research Group, University of Edinburgh, Edinburgh, UK; ${ }^{3}$ Heart Failure Nursing Service, NHS Lothian, Edinburgh, UK; ${ }^{4}$ University of Edinburgh, Edinburgh, UK; ${ }^{5}$ Department of Cardiology, University of Edinburgh, School of Medicine and Veterinary Medicine, Edinburgh, UK

Introduction Heart failure has a worse survival rate than many common cancers, yet few patients receive any palliative care input during the course of their illness. One of the main difficulties in providing palliative care for heart failure patients is the uncertainty around the course of the disease and the patient's life expectancy. The aim of this study was to compare the "Gold Standards Framework" (GSF) criteria, which were developed to determine the need for palliative care in non-cancer patients, with the "Seattle Heart Failure (SHF) Model", which provides a method of calculating a patient's predicted mean life expectancy using physiological variables.

Methods Chronic heart failure patients, in NYHA class III or IV, who were being managed in the specialist, heart failure nursing service, were identified from a clinical heart failure database. GSF criteria were assessed by interviewing the specialist nurse responsible for each patient's care. Clinical data required for the SHF model were obtained from two, online databases and were used to estimate mean life expectancy and predicted mortality at 1 year. Patients were then followed up, at 1 year, to evaluate; 1) all cause mortality, 2) place of death, and 3) the sensitivity and specificity of the GSF and SHF to predict death at 1 year.

Results 138 NYHA III-IV patients were identified from a total of 368 patients currently managed within the specialist nurse service; $66 \%$ were male, and the mean age was 77 years. GSF criteria, 